\title{
Assessment of the Need to Create Control Sytem of Unmanned Dump Truck
}

\author{
Dmitry Dubinkin ${ }^{1, *}$, Vladimir Sadovets ${ }^{1}$, Ilya Syrkin $^{1}$, and Ivan Chicherin ${ }^{1}$ \\ ${ }^{1}$ T.F. Gorbachev Kuzbass State Technical University, 28, Vesennyaya street, Kemerovo, 650000 , \\ Russian Federation
}

\begin{abstract}
The paper substantiates the need to create an autonomous dump truck control system. The scope of the unmanned mining dump truck is described. An example of the layout of the general scheme of an autonomous dump truck with control system basic elements is given, as well as some differences from the dump trucks with traditional control system are presented. The levels of automation of autonomous dump truck are formed. The enlarged tasks that need to be addressed when creating a control system for autonomous mining dump truck are highlighted. The general structure of a multi-level control system for autonomous dump truck was developed. The upper, middle and lower levels of the control system are described. The summation is drawn on the relevance of developing scientifically based approaches for creating control systems for autonomous mining truck, as well as developing mathematical models and algorithms for controlling autonomous mining trucks. The main directions for further research are identified. They are the development of requirements for a dump truck control system; the development of the structure of mathematical models and algorithms for the operation of subsystems of the dump truck control; the development of software that allows simulating the interaction of the subsystems of the dump truck control system with each other, the Smart Quarry system and the operator.
\end{abstract}

\section{Introduction}

Currently, unmanned vehicles are widely used in various fields of human activity. The use of unmanned vehicles in industry is dictated by harmful and dangerous working conditions, as well as the need to increase the labor productivity.

The use of unmanned vehicles in cargo moving avoids driver errors caused by fatigue, limited visibility, etc. Limitation of the expansion of autonomous vehicles is related to the cost of equipment providing autonomous work of vehicles [1-4].

This article discusses technical solutions for the organization of autonomous vehicle control system, which solve the problems of rock mass transporting at open pit mining.

The Russian company "VIST Robotics", as the part of the Intelligent Quarry Project, has developed the dump truck automated control system that allows excluding a man from dangerous conditions and moving him/her to more comfortable conditions [5-7]. The main

\footnotetext{
* Corresponding author: ddm.tm@kuzstu.ru
} 
advantages of the system are the following: operator safety; possibility for one operator to control 4-10 dump trucks; $20 \%$ performance improvement; possibility of mining in hard-toreach and climate-intensive regions.

Together with BELAZ, a prototype of an unmanned dump truck based on the BelAZ7513R model was created (Fig. 1). The dump truck can operate in both offline and telemetric control modes.

BelAZ-7513R operates as follows: the dispatcher provides the system with the assignment for the shift, allocates the required number of equipment units and the production route. Dump trucks leave the parking lot for the quarry site and start a continuous work loading, moving along the road, and unloading. The operator can reassign the vehicle to the second route or send it to the parking lot: the robot will finish the current cycle and go to a new task $[8,9]$.

In case of difficult conditions, remote control is taken over by the operator (Fig. 2), who can control several vehicles at the same time.

Autonomous control system of dump truck (BelAZ-7513R) movement (Fig. 1) includes on-board control box located in front part of vehicle near driver's cabin. Two receivers of satellite navigation systems are installed on left and right sides of the vehicle [10].

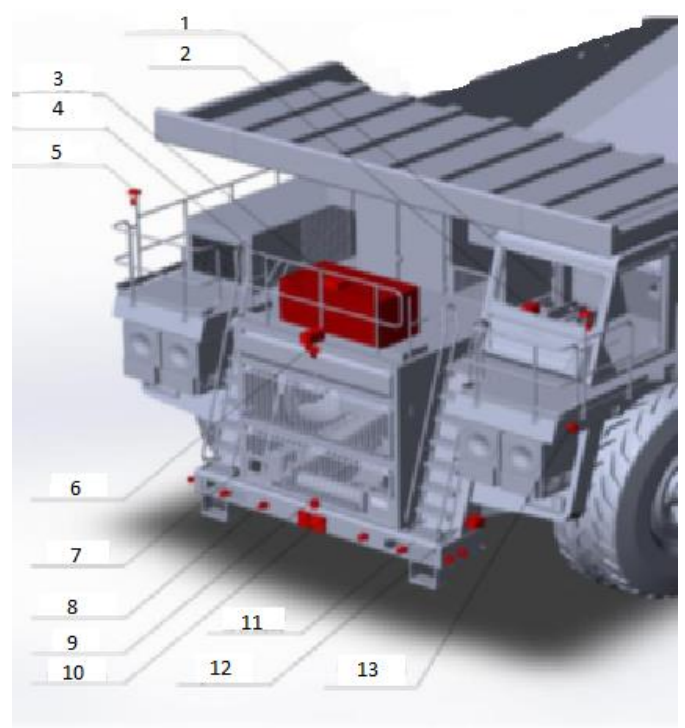

Fig. 1. The main elements of an autonomous control system of the dump truck movement (BELAZ7513R): 1) GPS receiver; 2) information panel; 3) power box monitoring unit; 4) power box; 5) GPS receiver; 6) camera; 7) motion sensor; 8) parctronic; 9) radar; 10) lidar; 11) parctronic; 12) camera; 13) round-view camera. 


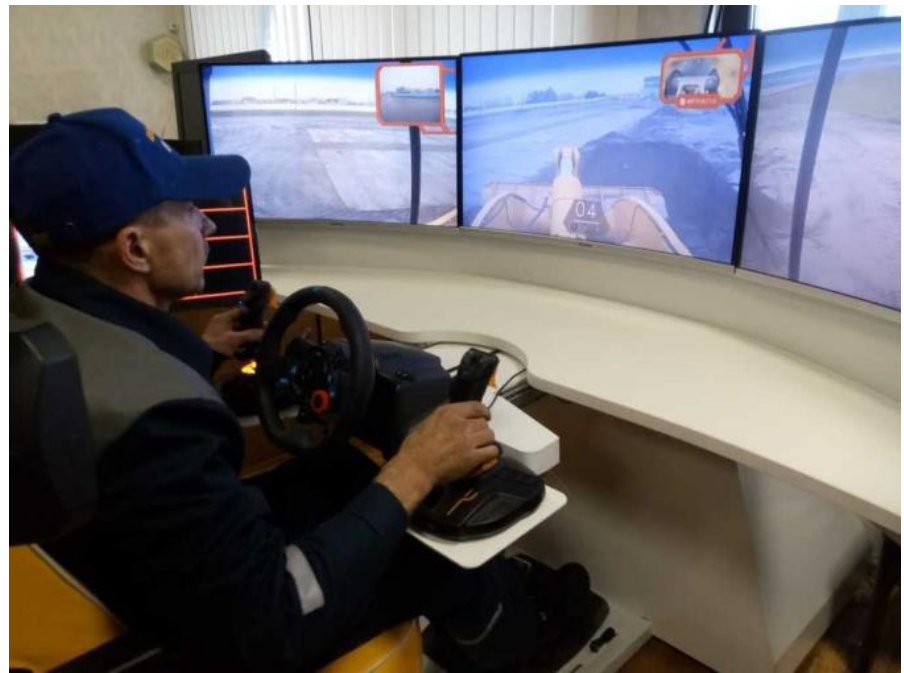

Fig. 2. Remote workplace of a driver

For visual control, 11 video cameras are used, which are installed around the perimeter of the vehicle [11]. The car uses Orlaco EMOS series protected cameras with an Ethernet interface. Several Delphi ESR radars operating in the medium and long range are responsible for detecting people and other objects along the route.

In case of difficult conditions, the operator takes over the remote control (Fig. 2), and can simultaneously control several vehicles at once.

The autonomous traffic control system of the BELAZ-7513R dump truck (Fig. 1) includes an onboard control box located in front of the car, next to the driver's cab. On the left and right sides of the car two receivers of satellite navigation systems were installed [10].

In the long-range mode, the viewing angle is $+/-10$ degrees, the range is $174 \mathrm{~m}$. In the medium-range mode, the viewing angle is $+/-45$ degrees, the range is $60 \mathrm{~m}$. Radar data update period is $50 \mathrm{~ms}$. Quanergy 3D lidar is installed in front of the car. Mark VIII QUA-M8 lidar allows $360^{\circ}$ scanning in the horizontal plane and $20^{\circ}$ in the vertical. The lidar works in the range of $1 \div 150 \mathrm{~m}$ and is used for three-dimensional mapping.

In addition to the three-dimensional lidar, two-dimensional Leddar IS16 lidars from LeddarTech are used. IS16 is an industrial solid-body lidar without moving parts and it is used to control the safety zone around the car. Lidar has a viewing angle of 45 degrees, divided into 16 segments. The range of lidar is $50 \mathrm{~m}[12,13]$.

Along the perimeter of the vehicle ultrasonic sensors (sonars) Banner QT50U are installed. Ultrasonic sensors are used to detect obstacles at small locations, the detection range of the sensor is $0.2-8.0 \mathrm{~m}$ [14.15].

Companies such as Komatsu, Volvo, SCANIA, Caterpillar and others have been developing autonomous control systems for dump trucks for a long time.

\section{Results and Discussion}

To ensure the effective operation of an autonomous dump truck, it is necessary to develop a control system that implements many tasks. The list of tasks that are solved when driving an autonomous dump truck depends on the technical characteristics of a particular dump truck model. This is especially important when developing and creating a new type of dump truck, because at the stage of constructive solutions formation there is the possibility of adapting the elements of autonomous control system to the developing construction of a dump truck. 
The tasks associated with the development of autonomous control system for a dump truck can be divided into the following classes:

- traffic control of autonomous dump truck along the road;

- improving the efficiency of autonomous dump truck;

- positioning of autonomous dump truck in space;

- controlling of auxiliary systems of a mining truck;

- diagnostics of the state of autonomous dump truck;

- interaction of the control system of autonomous dump truck with an operator;

- interaction of the control system of autonomous dump truck with a dispatch point;

- interaction of the control system of autonomous dump truck with other devices included in the Smart Quarry system.

The task of controlling the movement of autonomous mining truck, in the first approximation, is reduced to the task of controlling the actuators, the main of which are gear motors, steering and brake system. The wheels are driven by the gear motor, and the steering system of autonomous dump truck provides maneuvers along the road. The solution to this problem can be carried out both in manual and automatic modes. In manual mode, the operator, using the controls, turns on the actuators and changes their operating parameters for maneuvering and changing the speed of autonomous dump truck. In automatic mode, the automated dispatching system sets the trajectory and speed of movement, and the autonomous control system of the dump truck calculates the parameters of the actuators and gives them the corresponding control actions.

The task of improving the performance of autonomous dump truck is divided into two subtasks:

- improving the performance of autonomous dump truck based on optimization criteria;

- improving the performance of autonomous mining truck based on the use of adaptive algorithms that take into account external factors, such as the features of the open pit mine and the dynamic parameters of the dump truck itself.

To solve the optimization problem, it is necessary to develop mathematical models and intelligent algorithms for the operation of control system of autonomous dump truck, taking into account the interconnection of all its elements. These models and algorithms will make it possible to apply control actions to the actuators under which the operation of autonomous dump truck will satisfy the specified optimization criterion. Optimization criteria can be the energy consumption of autonomous dump truck per unit path, the accuracy of the trajectory of movement, etc.

Currently, the task of orientation and navigation in the space of ground objects is effectively solved using global positioning systems (GLONASS, GPS). However, for mining machines, the use of such systems is possible with other positioning systems based on inertial navigation systems, because sometimes in quarries satellite signals do not reach them. Thus, the development of a positioning system for autonomous dump truck in space is the subject of further research. The operation of such a system should be based on a combination of satellite navigation technologies, odometric sensors and inertial navigation.

The auxiliary systems of autonomous mining dump truck are the systems supplementing the main process - the movement of autonomous dump truck along the quarry route. Such systems include: power unit, vessel body dumping drive. The task of control over these fundamentally different systems should also be addressed by the control system of autonomous dump truck.

The heavy operating conditions of autonomous dump truck, as well as the high level of dynamic loads predetermine special requirements for its reliability. One of the important factors for improving reliability in operating conditions is technical diagnostics. The control system of autonomous dump truck should include a diagnostic subsystem that monitors the technical condition by measuring and displaying the parameters of elements of autonomous mining truck, 
automation devices, electrical and hydraulic systems, lubrication systems, bearing assemblies, etc.

The main technical means for the interaction of the control system of autonomous dump truck with the operator are the control panel and information display device. Using the control panel, the operator submits control actions to the subsystems, executive mechanisms and devices of autonomous dump truck operating in manual, semi-automatic (automatic) or automatic modes. In semi-automatic mode, it is necessary to solve the problem of a clear separation of control functions between the operator and the control device. The information display device (monitor) allows the operator to view the status of processes and elements, the presence of emergencies, the results of the diagnostic system in real time and retrospectively. It is also possible to implement additional sound and light alerts for the operator, for example, to signal emergencies. The task of the control system is to provide the operator with the necessary information.

In addition, for the effective operation of autonomous dump truck in conjunction with other mining machines, it is necessary to solve the problems of developing models for the interaction of autonomous dump truck control system with a control center.

Given the complexity and variety of formulated problems that are solved when driving an autonomous dump truck, as well as taking into account the complex structure of the construction of autonomous dump truck, consisting of many interconnected systems, the general structure of a multilevel control system for autonomous dump truck was developed, which is presented on Fig. 3.

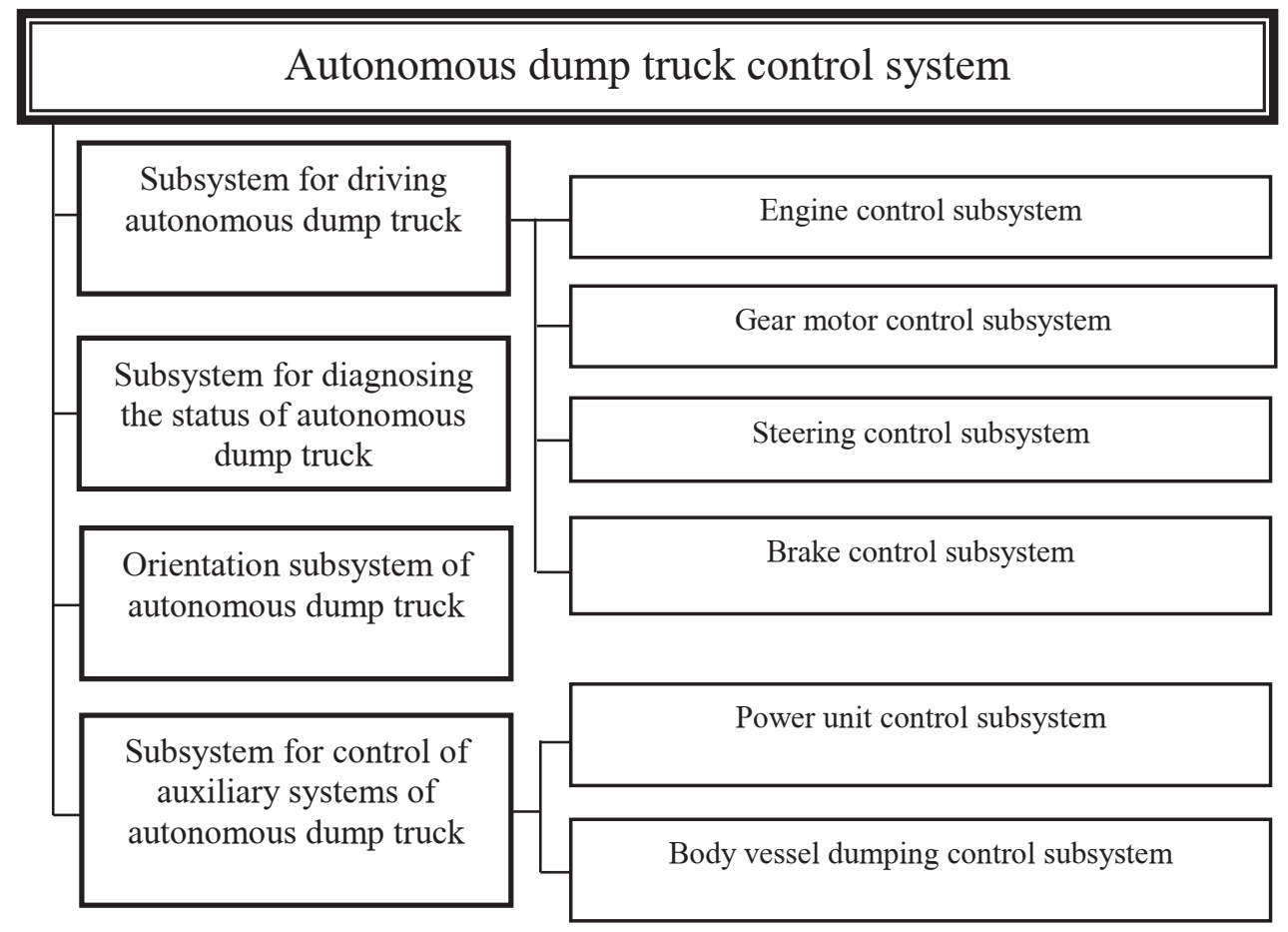

Fig. 3. General structure of autonomous dump truck control system

The top-level control system is an information management system that solves the tasks of coordinating the work of downstream subsystems, exchanging information between these subsystems, as well as interacting autonomous dump truck control system with an operator and 
a dispatch center, diagnosing the status and orientation of autonomous dump truck in space. The middle level of the control system is represented by drive control subsystems. The lower level is represented by the control subsystems of the drives of the main and auxiliary systems (engine, gear motors, brake system, steering system, vessel body dumping device, etc.).

\section{Conclusion}

The presented tasks and the general structure of the control system allows concluding that the tasks solved by the control system of autonomous dump truck are complex and diverse. However, currently there are no scientifically based approaches for development, mathematical modelling and algorithms drawing of control systems for autonomous dump truck. This hinders the creation of autonomous mining truck with fundamentally new characteristics. Therefore, studies aimed at solving these problems are relevant.

To continue research, it is necessary to do the following:

- to formulate the requirements for the control system of autonomous dump truck;

- to analyze the tasks solved by the control system of autonomous dump truck;

- to develop the structure, mathematical models and operation algorithms of the allocated subsystems of the control system of autonomous dump truck;

- to develop equipment requirements for autonomous dump truck control system;

- to create a list of necessary equipment to ensure autonomous control of dump truck;

- to develop software that allows simulating the interaction of the subsystems of control system of autonomous mining truck with each other;

- to develop software that allows simulating the interaction of control subsystems of autonomous dump truck with the Smart Quarry system.

\section{Acknowledgement}

The research was financially supported by the Ministry of Science and Higher Education of the Russian Federation in the framework of agreement No. 075-11-20 19-034 of November 22, 2019. with PJSC "KAMAZ" on the integrated project "Development and creation of high-tech production of autonomous heavy platforms for unattended mining in the Smart Quarry" system, with the participation of the Federal State Budget Educational Establishment of Higher Education T.F. Gorbachev Kuzbass State Technical University "in terms of the implementation of research, development and technological work.

\section{References}

1. K. N. Trubetskoy, D. A. Klebanov, S. V. Yasyuchenya, Gornyy zhurnal, 10, 67 (2013)

2. I. A. Sokolov, A. S. Misharin, V. P. Kupriyanovskiy, O. N. Pokusaev, O. N. Larin, International Journal of Open Information Technologies, 6, 44 (2018)

3. S. Fiscor, Engineering \& Mining Journal, 11, 52 (2018)

4. A. Kuchumova, Dobyvayushchaya promyshlennost, 2, 92 (2019)

5. VIST Grupp i SUEK ispytyvayut bespilotnye BELAZy na razreze v Khaka-sii. - Rezhim dostupa: https://vistgroup.ru/media/news/nid/vist-group-and-suek-are-unmanned-belazon-mine-in-khakassia/

6. A. S. Baranova, A. E. Okhrimenko, A. P. Stolyarova, N. A. Stenina, Sbornik materialov IX Vserossiyskoy nauchno-prakticheskoy konferentsii molodykh uchenykh $s$ mezhdunarodnym uchastiem "Rossiya molodaya", 39 (2017).

7. Yu. Voronov, A. Voronov, S. Grishin, A. Bujankin, E3S Web of Conferences The Second Inter-national Innovative Mining Symposium (2017) 
8. G. D. Buyalich, A. S. Furman, Gornoe oborudovanie i elektromekhanika, 5, 40 (2017)

9. S. S. Shadrin, O. O. Varlamov, A. M. Ivanov, Journal of Advanced Transportation, 10 (2017) doi:10.1155/2017/2492765.

10. V. Nesterov, V. Aksenov, V. Sadovets, D. Pashkov, E3S Web of Conferences IVth International Innovative Mining Symposium 03001, (2019)

11. D. V. Stenin, Gornoe oborudovanie i elektromekhanika, 6, 3 (2019)

12. D. M. Dubinkin, Mining Equipment and Electromechanics, 6, 8 (2019).

13. V. Aksenov, I. Chicherin, I. Kostinez, A. Kazantsev, A. Efremenkov, E3S Web of Conferences The Second International Innovative Mining Symposium (2017)

14. A. S. Abroskin, Vestnik Irkutskogo gosudarstvennogo tekhnicheskogo universiteta, 10, $121(2014)$

15. V.V. Aksenov, A.B. Efremenkov, V.Yu. Sadovets, D.A. Pashkov, IOP Conference Series: Materials Science and Engineering, 012005 (2018) 